\title{
Randomized clinical trial on preoperative radiotherapy 25 Gy in rectal cancer-treatment results at 5-year follow-up
}

\author{
Radoslaw Pach • Jan Kulig • Piotr Richter • \\ Tomasz Gach • Miroslaw Szura $\cdot$ Teresa Kowalska
}

Received: 13 October 2011 / Accepted: 30 November 2011 / Published online: 15 December 2011

(C) The Author(s) 2011. This article is published with open access at Springerlink.com

\begin{abstract}
Purpose The purpose of this study was to establish the influence of time interval between preoperative hyperfractionated radiotherapy $(5 \times 5 \mathrm{~Gy})$ and surgery on long-term overall survival (5 years) and recurrence rate in patients with locally advanced rectal cancer operated on according to total mesorectal excision technique.

Methods The treatment group comprised 154 patients with locally advanced rectal cancer who were operated on between 1999 and 2006 in the 1st Department of General Surgery, Jagiellonian University, Cracow, Poland. The data on survival has been systematically collected until 31 st of December 2010. In addition, the following aspects were analyzed: the significance of time interval between the end of radiotherapy and surgical treatment and its influence on downsizing, downstaging, rate of curative resections, and sphincter-sparing procedures. Patients were qualified to preoperative radiotherapy $5 \times 5$ Gy and then randomly assigned to subgroups with different time intervals between radiotherapy and surgery: one subgroup consisted of 77 patients operated on 7-10 days after the end of irradiation, and the
\end{abstract}

R. Pach $(\bowtie) \cdot$ J. Kulig $\cdot$ T. Gach $\cdot$ M. Szura

1st Department of General Surgery, Jagiellonian University, Cracow, Poland

e-mail: radoslaw.pach@uj.edu.pl

P. Richter

3rd Department of General Surgery, Jagiellonian University, Cracow, Poland

T. Kowalska

Department of Teleradiotherapy, Oncological Centre,

Cracow, Poland second subgroup consisted of 77 patients operated on after 4-5 weeks. Both groups were homogenous in sex, age, cancer stage and localization, distal and circumferential resection margins, and number of resected lymph nodes. Results The 5-year survival rate in patients operated on 7 10 days after irradiation was $63 \%$, whereas in those operated on after 4-5 weeks, it was $73 \%$ - the difference was not statistically significant (log rank, $p=0.24$ ). A statistically significant increase in 5-year survival rate was observed only in patients with downstaging after radiotherapy $-90 \%$ in comparison with $60 \%$ in patients without response to neoadjuvant treatment (log rank, $p=0.004$ ). Recurrence was diagnosed in $13.2 \%$ of patients. A lower rate of systemic recurrence was observed in patients operated on 4-5 weeks after the end of irradiation $(2.8 \%$ vs. $12.3 \%$ in the subgroup with a shorter interval, $p=0.035$ ). No differences in local recurrence rates were observed in both subgroups of irradiated patients $(p=$ $0.119)$. The longer time interval between radiotherapy and surgery resulted in higher downstaging rate $(44.2 \%$ vs. $13 \%$ in patients with a shorter interval, $p=0.0001$ ) although it did not increase the rate of sphincter-saving procedures $(p=0.627)$ and curative resections $(p=0.132)$.

\section{Conclusions}

1. Improved 5-year survival rate is observed only in patients with downstaging after preoperative irradiation dose of $25 \mathrm{~Gy}$.

2. Longer time interval after preoperative radiotherapy 25 Gy does not improve the rate of sphincter-saving procedures and curative resections (R0) despite higher downstaging rate observed in this regimen.

Keywords Rectal cancer - Radiotherapy - Overall survival . Recurrence rate 


\section{Introduction}

Colorectal cancer is a frequent neoplasm in both sexes. Approximately $40 \%$ of all colorectal cancers are diagnosed in the rectum. Surgery still remains the cornerstone of rectal cancer treatment. Over recent decades, surgical therapy of rectal cancer has witnessed a variety of developments until present surgical quality standards could be achieved. Preoperative irradiation is reported to have less morbidity and be more effective than postoperative regimens [1]. Two most common regimens of preoperative radiotherapy exist. The European model includes hyperfractionated treatment applied during 5 days (a total dose of 25 Gy) and operative treatment during a week after the completion of the scheme. The short delay is recognized to minimize postoperative complications. The American model comprises chemoradiotherapy (45-50 Gy +5-fluorouracil) and operative treatment after $4-8$ weeks. The literature is inconclusive regarding the real value of preoperative irradiation and optimal time interval before surgery has not been unequivocally established so far. Currently, there are no enough randomized trials that have reported whether there is a significant difference in overall survival, recurrence rates, tumor downstaging, or rate of sphincter-preserving procedures between these two neoadjuvant therapy schedules. Surprisingly, data for the association between time interval after preoperative radiotherapy and long-term outcomes are scarce as well. The influence of radiotherapy on survival was reported for the first time in 1997 by Swedish scientists (Swedish Rectal Cancer Trial), but potential flaws in this report (total mesorectal excision [TME] was not uniformly controlled) preclude a reliable evaluation of data [2]. Other authors diminish the role of radiotherapy particularly after introducing the TME technique, which enables better local clearance and thus results in a lower rate of locoregional recurrences [3]. The purpose of this randomized study was to ascertain the relationship between time interval after radiotherapy and long-term recurrence rate and overall survival of patients undergoing resection for locally advanced rectal cancer after neoadjuvant radiotherapy at a dose of $25 \mathrm{~Gy}$.

\section{Materials and methods}

\section{Patient selection}

All patients with locally advanced rectal cancer after neoadjuvant radiotherapy 25 Gy operated on between 1999 and 2006 at the $I^{\text {st }}$ Department of General Surgery in Krakow were included in the study. The treatment group consisted of 154 patients who were qualified to preoperative radiotherapy $5 \times 5$ Gy and then randomly assigned to subgroups with different time intervals between radiotherapy and surgery: one subgroup consisted of 77 patients operated on 7-10 days after the end of irradiation, and the second subgroup consisted of 77 patients operated on after 4-5 weeks. Computer-generated randomization tables were used.

All relevant data including demographics, clinical and histological staging, and details of diagnostic and surgical procedures were collected prospectively using a standard electronic database.

This study was approved by the Bioethics Committee of the Jagiellonian University and has been performed in accordance with the ethical standards laid down in the Declaration of Helsinki. All patients gave their informed consent prior to their inclusion in the study.

\section{Treatment and follow-up}

Preoperative irradiation was conducted at the Teleradiotherapy Department, Oncological Centre, Cracow, Poland. It comprised five doses of $5 \mathrm{~Gy}$ given for 5 consecutive days. Threeportal technique, three-dimensional planning, and an energy of 6 or $18 \mathrm{MV}$ were used. The accelerators Clinac 600 and Clinac 2300 were produced by Varian Medical Systems. The dose was calculated according to ICRU 50 and ICRU 62 guidelines. Inconsistency of the dose distribution in the irradiated area varied from $-5 \%$ to $5 \%$. The surgical treatment included TME with D3-lymphadenectomy, which followed the recommendations of the Japanese Society for Cancer of the Colon and Rectum. Anterior resection was performed if a 2-cm margin below tumor was possible to achieve. In other cases, abdominoperineal resection was performed. Only few patients underwent Hartmann procedure or local excision (TEM). Follow-up data were collected based on clinical examination every 3 to 6 months after discharge, and dates of death were verified using data obtained from the census registry office. Survival rates were calculated based on the overall survival principle; that is, deaths due to any cause were accepted as complete observations, while lost to follow-up was considered as a censored observation. Recurrence rates were calculated only for patients who underwent potentially curative resections (R0).

Statistical methods

Mann-Whitney $U$ and $\chi^{2}$ tests were used where appropriate to compare the distribution of individual variables between groups. Survival data were analyzed according to the Kaplan-Meier method and included postoperative mortality; the log-rank test was used to detect differences between groups. $p<0.050$ was considered statistically significant. Statistical analyses were performed using the Statistica version 9.0 (Stat Soft Inc.) software package. The local and systemic recurrence rate for preoperative radiotherapy and early surgery was estimated at approximately $14 \%$ at 5 years, whereas the 
local and systemic recurrence rate for delayed surgery was estimated as $2 \%$. Sample Power release 2.0 (SPSS Inc.) was used to calculate the population required to achieve a test power of $80 \%$. Sample size needed for each arm was established as 80 patients (two-sided test, $\alpha=0.05$ ). The actual recruitment in the study was slightly lower (77 patients in both arms), so the actual power is lower than $80 \%$. The study was registered at ClinicalTrials.gov, identifier NCT01444495.

\section{Results}

Demographic data

Between 1999 and 2006, 154 patients were operated on after neoadjuvant radiotherapy $5 \times 5$ Gy. Table 1 shows the clinicopathological features of the analyzed population. Both analyzed groups were homogenous for age, sex, clinical staging, tumor localization, and resection margins (Table 1).

\section{Surgery}

No significant differences between the analyzed groups were observed for the type of resection, number of resected lymph nodes, numbers of patients with at least 12 lymph nodes retrieved, rates of potentially curative resections, and rates of sphincter-saving procedures. The procedure of choice was TME. All operations were performed by the same team of surgeons trained in TME technique. D3lymphadenectomy was conducted as a standard procedure; that is, mesorectal, inferior mesenteric and periaortic lymph nodes to the level of pancreas were resected.

\section{Recurrences}

The recurrence rates altogether did not differ between the analyzed groups. If specific types of recurrences were evaluated separately, more systemic recurrences were observed in patients operated on after a short time interval between the end of irradiation and surgery $(12.3 \%$ vs. $2.8 \%$, respectively; $p=0.035)$. Rates of local recurrences did not differ either $(p=0.119)$. The exact numbers and percentages of recurrences are summarized in Table 2.

\section{Long-term survival}

Until the final follow-up (on December 31, 2010), 57 of 154 patients had died, and the median follow-up period for those who survived was 86 months. The overall survival in patients operated on after different time intervals between radiotherapy and surgery was similar (Fig. 1). Elongation of time interval did not improve the survival rate. Survival benefit was observed only in patients who responded to preoperative treatment, that is, when downstaging was confirmed (Fig. 2). In this subgroup, 5 -year overall survival was significantly higher than in the patients who did not respond to radiotherapy $(90 \%$ vs. $60 \%$, respectively; $p=0.004$ ).

\section{Downstaging}

Staging of rectal cancer was performed according to the Union for International Cancer Control/American Joint Committee of Cancer (UICC/AJCC) 2002. Clinical stage of the neoplasm was assessed in preoperative examinations performed before radiotherapy: endorectal ultrasound, abdominal ultrasound, and chest $x$-ray. Pathological TNM (ypTNM) was determined by a histologist after examination of the specimen. Downstaging was recorded when pathological stage was lower than clinical stage before neoadjuvant treatment. A change only in T feature without change in cancer stage was not regarded as downstaging in this study. Complete pathological response was defined as the absence of a residual tumor at the time of histological examination of the resected specimen. Higher rates of patients responding to radiotherapy were observed after a longer delay between irradiation and surgery $(p=0.0001)$. Complete responses were observed only after a longer time interval in $10.4 \%$ of patients.

\section{Discussion}

The influence of preoperative radiotherapy on survival

Hyperfractionated radiotherapy and chemoradiotherapy have been the principal neoadjuvant regimens assessed in randomized trials on combined treatment of rectal cancer. Short-course preoperative irradiation (a total dose of 25 Gy divided into five fractions) was reported to have a survival benefit in a Swedish Rectal Cancer Trial [2]. In that study, patients were assigned to 1-week hyperfractionated radiotherapy followed by surgery within 7 days versus surgery alone. The 5-year overall survival rate was $58 \%$ in the combined treatment group compared with $48 \%$ in the surgery-alone group $(p=0.004)$. One flaw of the study is that it was conducted prior to the introduction of TME as the standard procedure. Furthermore, the study did not comprise only patients with locally advanced neoplasm, as both arms included subjects with Dukes A disease.

In another randomized study by Bujko et al. [4] (Polish Trial), patients given chemoradiation compared with those who underwent radiation alone $(5 \times 5 \mathrm{~Gy})$ had no significant difference in 4-year survival (66\% vs. $67 \%$, respectively). The Dutch Rectal Cancer Study Group conducted a randomized trial to determine whether neoadjuvant radiation offers any benefit to patients who uniformly undergo TME. Unlike previously mentioned Swedish Trial, this study has not shown a difference in overall survival [5]. Different lengths 
Table 1 Patient characteristics in analyzed groups

Preoperative radiotherapy 25 Gy + surgery, $n=154$

Time interval 7-10 days, $n=7 \quad$ Time interval $4-5$ weeks, $n=77$

Age (years), median (range)

Women

Men

Clinical staging of rectal cancer according to UICC (AJCC 2002)

I

II

III

Tumor localization (distance from anal verge)

$<6 \mathrm{~cm}$

$7-12 \mathrm{~cm}$

Mean resection margins $(\mathrm{cm})$

Proximal

Distal

Circumferential (radial)

Resected lymph nodes

Median (range)

Patients with $\geq 12$ lymph nodes resected

Adjuvant chemotherapy

No. of patients $(\%)$

Types of resection

Anterior resection

Abdominoperineal amputation

Hartmann procedure

TEM

Other (hemicolectomy + anterior resection)

Type of resection according to Hermanek

R0

R1

$\mathrm{R} 2$

Sphincter-saving operations

No. (\%)

Response to radiotherapy

Downstaging

Complete response

Histological staging after radiotherapy (ypTNM, AJCC 2002)

0

I

II

III

IV

$$
\begin{array}{r}
61(30-92) \\
28(36.4 \%) \\
49(63.6 \%) \\
9(11.7 \%) \\
45(58.4 \%) \\
23(29.9 \%) \\
28(36.4 \%) \\
49(63.6 \%)
\end{array}
$$

$15.83 \pm 7.27$

$4.30 \pm 1.89$

$1.11 \pm 1.1$

$16(1-79)$

$56(73 \%)$

$53(68.8)$

$40(51.9 \%)$

$31(20.1 \%)$

$5(6.5 \%)$

0

$1(1.3 \%)$

$$
\begin{gathered}
65(84.4 \%) \\
7(9.1 \%) \\
5(6.5 \%)
\end{gathered}
$$

41 (53.2)

$10(13 \%)$

0

$$
\begin{gathered}
1(1.3 \%) \\
20(26 \%) \\
18(23.4 \%) \\
34(44.1 \%) \\
4(5.2 \%)
\end{gathered}
$$

$0.87^{\mathrm{a}}$

$0.10^{\mathrm{b}}$

$38(49.4 \%)$

$39(50.6 \%)$

$14(18.2 \%)$

$44(57.1 \%)$

$19(24.7 \%)$

$37(48.1 \%)$

$40(51.9 \%)$

$0.14^{\mathrm{b}}$

$14.67 \pm 6.30$

$0.303^{\mathrm{a}}$

$4.20 \pm 2.36$

$0.778^{\mathrm{a}}$

$0.618^{\mathrm{a}}$

$1.27 \pm 1.13$

$0.204^{\mathrm{a}}$

$15(0-57)$

$0.382^{\mathrm{b}}$

$51(66 \%)$

$0.731^{\mathrm{b}}$

$51(66.2)$

$0.391^{\mathrm{b}}$

25 (16.2\%)

8 (10.4\%)

$4(5.2 \%)$

0

$$
\begin{gathered}
71(92.2 \%) \\
2(2.6 \%) \\
4(5.2 \%)
\end{gathered}
$$

44 (57.1)

$0.627^{\mathrm{b}}$

$34(44.2 \%)$

$0.0001^{\mathrm{b}}$

$8(10.4 \%)$

$0.003^{\mathrm{b}}$

$11(14.2 \%)$

$0.003^{\mathrm{b}}$

$25(32.5 \%)$

23 (29.9\%)

15 (19.5\%)

3 (3.9\%)

${ }^{\text {a } M a n n-W h i t n e y ~} U$ test

${ }^{\mathrm{b}} \chi^{2}$ Test

of follow-up, 2 years in the Dutch Trial and 5 years in the Swedish Trial, might be a substantial factor contributing to this inconsistency. The latest update of the Dutch Trial published in 2007 revealed no significant difference in a 5-year overall survival either [6].
Our study is one of the first randomized trials where long-term treatment results are summarized for the patients operated on after a longer time interval following the preoperative hyperfractionated radiotherapy $25 \mathrm{~Gy}$. Delay of surgery (4-5 weeks after radiation) did not improve 


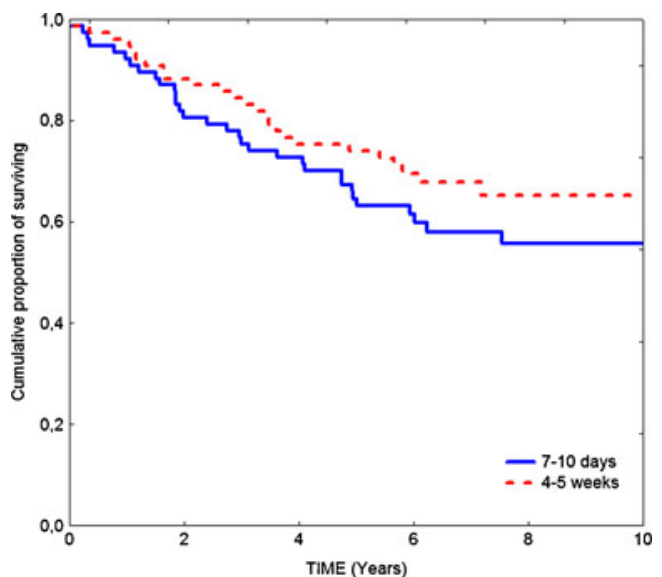

Fig. 1 Overall survival in patients operated on after 7-10 days and 45 weeks after preoperative radiotherapy 25 Gy (5-year survival rate, $63 \%$ vs. $73 \%$; log rank, $p=0.24$ )

the 5-year overall survival either. Patients probably benefited mainly from the high quality surgery (TME was uniformly controlled, and more than $70 \%$ of patients had at least 12 lymph nodes retrieved), and despite a higher downstaging rate, no improvement in survival was achieved.

There are few ongoing trials that address the issue of optimal time interval before surgery. The Stockholm III Trial is examining the different preoperative radiotherapy regimens and delay to surgery - it is comparing $5 \times 5$-Gy radiotherapy and immediate surgery versus $5 \times 5$-Gy and delayed surgery versus 50 -Gy radiotherapy and delayed surgery [7]. The study purpose is to determine whether the timing of surgery after radiation influences morbidity, mortality, and tumor downstaging. Unfortunately, the results of this trial have not been published so far.

In our study, a significant survival benefit was observed in patients who responded to radiotherapy; that is, when downstaging was confirmed (5-year overall survival of $90 \%$ in comparison with $60 \%$ in the patients without response to

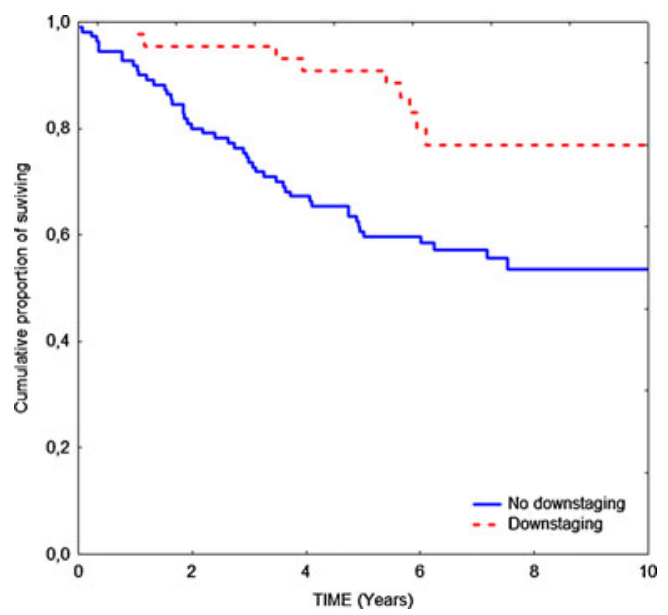

Fig. 2 Overall survival in patients with and without downstaging after preoperative radiotherapy 25 Gy (5-year survival rate, $90 \%$ vs. $60 \%$; $\log$ rank, $p=0.004$ )
Table 2 Pattern of recurrences in analyzed groups

\begin{tabular}{lccl}
\hline & $\begin{array}{l}\text { Time interval } \\
7-10 \text { days, } n=77\end{array}$ & $\begin{array}{l}\text { Time interval } \\
\text { 4-5 weeks, } n=77\end{array}$ & $\mathrm{p}$ \\
\hline Local & $1(1.5 \%)$ & $5(7 \%)$ & 0.119 \\
Systemic & $8(12.3 \%)$ & $2(2.8 \%)$ & 0.035 \\
Local + systemic & 0 & $2(2.8 \%)$ & 0.271 \\
Total & $9(13.8 \%)$ & $9(12.7 \%)$ & 0.520 \\
\hline
\end{tabular}

neoadjuvant treatment; $\log$ rank $p=0.004)$. Clinical data supporting the importance of the downstaging and pathologic response to preoperative radiotherapy are still abundant. Stipa et al. [8], in a study conducted at Memorial Sloan-Kettering Cancer Centre, compared patients with locally advanced rectal cancer who achieved a pathologic complete response with those without difference between the clinical stage (determined in endorectal ultrasound) and the pathologic stage. Results showed a 5-year recurrence-free survival rate of $96 \%$ for patients achieving a complete response in comparison with $54 \%$ in the nodownstaging group $(p<0.00001)$. This result is similar to the one observed in our research.

Although in our study, improvement of 5-year survival was observed in the patients with successful downstaging and tumor shrinkage was higher in patients allocated to delayed surgery, the survival of the whole collective was not significantly improved. This phenomenon might result from the fact that in some patients who did not respond to radiotherapy, delay in operative treatment led to tumor progression. Indeed, upstaging was observed in 8 patients (10.4\%) assigned to a long-interval arm, and these individuals were obviously included in the survival analysis. Even if progression was not apparently noticed in nonresponders, they were probably more liable to local or systemic recurrence, which resulted in poorer prognosis.

\section{Influence of preoperative radiotherapy on recurrences}

The risk of locoregional relapse of rectal cancer is affected by involvement of the circumferential (radial) resection margin and lymph node status. The conventional treatment in most countries for clinical stage T3 or node-positive rectal cancer is preoperative treatment, since a significantly lower local recurrence rate and morbidity were reported after preoperative than postoperative chemoradiation in the German Chirurgische Arbeitsgemeinschaft für Onkologie/Arbeitsgemeinschaft Radiologische Onkologie/Arbeitsgemeinschaft Internistische Onkologie (CAO/ARO/AIO-94) trial [1].

In Swedish Rectal Cancer Trial, the local recurrence rate at a 5 -year follow-up was $11 \%$ in the group receiving radiotherapy prior to surgery and $27 \%$ in the group treated with surgery alone $(p<0.001)$ [2]. Bujko et al. [4] reported no significant difference in local relapse for the patients who underwent different preoperative treatment schemes: chemoradiation or radiotherapy 
25 Gy (14\% vs. 9\%, respectively). The Dutch Trial confirmed results of the Swedish group, as they reported local recurrence rate of $2.4 \%$ at the 2 -year follow-up in the radiotherapy and surgery groups and $8.2 \%$ rate in the surgery-alone group ( $p<$ 0.001 ) [5]. The update of the trial published in 2007 revealed a reduction in local failure from $21 \%$ to $11 \%$ for stage III patients but no significant reduction for stage II patients [6]. In some respect, the promising results of TME challenged the advantages of preoperative irradiation. A Dutch TME Trial, however, proved that neoadjuvant radiation reduced the local recurrence rate also after TME [5, 6]. Furthermore, the Medical Research Council CR07 trial (MRC CR07) showed that postoperative chemoradiation for patients with positive circumferential margin does not compensate for the beneficial effect of the routine use of the short-course irradiation for all rectal cancer patients. In this trial, 1,350 patients were randomized to TME preceded by 25 Gy in 1 week versus TME followed by chemoradiation if the circumferential margin was positive. The rate of local recurrence at 5 years was significantly better in the preoperative irradiation group (5\%) compared with the postoperative radiotherapy group $(17 \%)(p<0.001)$. In multivariate analysis, the status of radial margin was the strongest predictive factor for local relapse of rectal cancer [9].

The hyperfractionated radiotherapy $5 \times 5$ Gy sterilizes the surgical margins of mesorectum and prevents the growth of residual foci of neoplastic cells. The oncological sterilization results in a lower rate of locoregional failure and may potentially decrease distant spread of tumor cells. This mechanism may explain the lower rate of distant metastases detected in our research in patients operated on 4-5 weeks after the end of radiotherapy. However, no difference in local relapse was observed in groups with different time intervals before surgery. In previously published studies, the short scheme of radiotherapy did not downstage the tumor when the time interval before operation was short $[10,11]$. On the contrary, in our study, $13 \%$ downstaging rate was observed in the short-interval group. This discrepancy may result from different timing of surgery: in our research, patients were operated on 7-10 days after the end of radiotherapy, whereas in other studies, the operation was scheduled usually earlier (before 7th day).

Despite discrepancies in overall survival, the majority of studies consistently showed that preoperative radiation therapy provides a significant reduction in local recurrence for locally advanced rectal cancers.

An ongoing trial by Garcia-Aguilar et al. is investigating whether locally advanced rectal cancer response rates are influenced by the delay of surgery after neoadjuvant chemoradiotherapy. Preoperative treatment consists of standard 5fluorouracil or the combination chemotherapy and radiotherapy 45 Gy given over a 6-week period. Surgical treatment is scheduled $6,10,14$, or 19 weeks after neoadjuvant treatment [7]. This trial will enable to determine the optimal time interval to surgery in respect to achieving the best long-term treatment results.

\section{Sphincter preservation}

Avoiding permanent stoma remains a crucial factor of quality of life. During our trial, the required distal margin of rectal cancer was at least $2 \mathrm{~cm}$ to perform the operation with sphincter preservation. Although a significantly higher rate of tumor shrinkage was achieved in the group with delayed surgery, it had no effect on lowering the rate of permanent stoma in these patients. This observation may be connected with applying a rule of 2-cm distal margin as well as surgeons' skills, patients' desire, and tumor localization. In addition, in some cases, tumor shrinkage was not sufficient to translate into a significant difference in the surgical approach. The issue of sphincter preservation was addressed by at least 17 randomized trials methodically reviewed by Gerard et al. [12]. They concluded that in none of the large trials, neoadjuvant treatment was able to reveal any significant benefit in terms of sphincter saving. Few randomized studies reported advantages of a particular preoperative therapy, but their results cannot be widely implemented into clinical practice due to the substantial flaws (low number of patients included, only nonresectable or recurrent tumors) [13, 14]. On the other hand, the reappraisal of the rule of the $2 \mathrm{~cm}$ for distal margin has increased the possibility of sphincter-saving surgery, which should be evaluated in further studies.

\section{Conclusions}

An improved 5-year overall survival rate is observed only in patients with downstaging after a preoperative radiotherapy dose of $25 \mathrm{~Gy}$. A longer time interval between radiotherapy and surgery increases the downstaging rate in patients with locally advanced rectal cancer. Preoperative radiotherapy 25 Gy does not improve the rate of sphincter-saving procedures and potentially curative resections (R0). Appropriately defining high-risk patients with advanced rectal cancer is crucial in providing neoadjuvant treatment only to those who would benefit mostly from irradiation.

Acknowledgements This research was conducted as a statutory work at the Jagiellonian University Medical College and Oncological Centre in Cracow, Poland. The authors declare that they have no conflicts of interest.

Conflicts of interest None.

Open Access This article is distributed under the terms of the Creative Commons Attribution Noncommercial License which permits any noncommercial use, distribution, and reproduction in any medium, provided the original author(s) and source are credited. 


\section{References}

1. Sauer R, Becker H, Hohenberger W et al (2004) Preoperative versus postoperative chemoradiotherapy for rectal cancer. N Engl J Med 351:1731-1740

2. Swedish Rectal Cancer Trial (1997) Improved survival with preoperative radiotherapy in resectable rectal cancer. N Engl J Med 336:980-987

3. MacFarlane JK, Ryall RDH, Heald RJ (1993) Mesorectal excision for rectal cancer. Lancet 341:457-460

4. Bujko K, Nowacki MP, Nasierowska-Guttmejer A, Michalski W, Bebenek M, Kryj M, for the Polish Colorectal Study Group (2006) Long term results of a randomized trial comparing preoperative short course radiotherapy with preoperative conventionally fractionated chemoradiation for rectal cancer. Br J Surg 93:1215-1223

5. Kapiteijn E, Marijnen CA, Nagtegaal ID et al (2001) Preoperative radiotherapy combined with total mesorectal excision for resectable rectal cancer. N Engl J Med 345(9):638-646

6. Peeters KC, Marijnen CA, Nagtegaal ID et al (2007) The TME trial after a median follow-up of 6 years: increased local control but no survival benefit in irradiated patients with resectable rectal carcinoma. Ann Surg 246(5):693-701

7. Julien LA, Thorson AG (2010) Current neoadjuvant strategies in rectal cancer. J Surg Oncol 101:321-326

8. Stipa F, Chessin DB, Shia J et al (2006) A pathologic complete response of rectal cancer to preoperative combined-modality ther- apy results in improved oncological outcome compared with those who achieve no downstaging on the basis of preoperative endorectal ultrasonography. Ann Surg Oncol 13(8):1047-1053

9. Sebag-Montefiore D, Stephens RJ, Steele R et al (2009) Preoperative radiotherapy versus selective postoperative chemoradiotherapy in patients with rectal cancer (MRC CR07 and NCIC-CTG C016): a multicentre, randomised trial. Lancet 373(9666):811-820

10. Taflampas P, Christodoulakis M, de Bree E, Melissas J, Tsiftsis DD (2010) Preoperative decision making for rectal cancer. Am J Surg 200(3):426-432

11. Marijnen CAM, Nagtegaal ID, Kranenbarg EK, Hermans J, van de Velde CJH, Leer JWH, van Krieken JHJM, for the Pathology Review Committee and the Cooperative Clinical Investigators (2001) No downstaging after short term preoperative radiotherapy in rectal cancer patients. J Clin Oncol 19(7):1976-1984

12. Gerard JP, Rostom Y, Gal J, Benchimol D, Ortholan C, Aschele C, Levi JM. Can we increase the chance of sphincter saving surgery in rectal cancer with neoadjuvant treatments: lessons from a systematic review of recent randomized trials. Crit Rev Oncol Hematol. 2011 Mar 4. [Epub ahead of print]

13. Gerard JP, Chapet O, Nemoz C et al (2004) Improved sphincter preservation in low rectal cancer with high-dose preoperative radiotherapy: the lyon R96-02 randomized trial. J Clin Oncol 22:2404-2409

14. Braendengen M, Tveit KM, Berglund A et al (2008) Randomized phase III study comparing preoperative radiotherapy with chemoradiotherapy in nonresectable rectal cancer. J Clin Oncol 26:3687-3694 